Please do not remove this page

RMIT

UNIVERSITY

\title{
Scaling of the space-time correlation function of particle currents in a suspensionof hard-sphere-like particles: Exposing when the motion of particles is brownian
}

Van Megen, William; Martinez, Vincent; Bryant, Gary

https://researchrepository.rmit.edu.au/esploro/outputs/9921864149701341/filesAndLinks?institution=61RMIT_INST\&index=null

Van Megen, W., Martinez, V., \& Bryant, G. (2009). Scaling of the space-time correlation function of particle currents in a suspensionof hard-sphere-like particles: Exposing when the motion of particles is brownian. Physical Review Letters, 103(25), 258302-1-258302-258304.

https://doi.org/10.1103/PhysRevLett.103.258302

Document Version: Accepted Manuscript

Published Version: https://doi.org/10.1103/PhysRevLett.103.258302

Repository homepage: https://researchrepository.rmit.edu.au

(c) 2009 The American Physical Society

Downloaded On 2023/04/26 19:25:25 +1000 
Thank you for downloading this document from the RMIT Research Repository.

The RMIT Research Repository is an open access database showcasing the research outputs of RMIT University researchers.

RMIT Research Repository: http://researchbank.rmit.edu.au/

\section{Citation:}

Van Megen, W, Martinez, V and Bryant, G 2009, 'Scaling of the space-time correlation function of particle currents in a suspension of hard-sphere-like particles: Exposing when the motion of particles is brownian', Physical Review Letters, vol. 103, no. 25, 258302, pp. $1-4$.

See this record in the RMIT Research Repository at:

http://researchbank.rmit.edu.au/view/rmit:5463

Version: Accepted Manuscript

Copyright Statement: ㄷ 2009 The American Physical Society

Link to Published Version:

http://dx.doi.org/10.1103/PhysRevLett.103.258302 


\title{
Scaling of the current-current time correlation function of a suspension of hard sphere-like particles: exposing when the motion of particles is Brownian.
}

\author{
W. van Megen, ${ }^{1}$ V. A. Martinez, ${ }^{1}$ and G. Bryant ${ }^{1}$ \\ ${ }^{1}$ Department of Applied Physics, Royal Melbourne Institute of Technology, Melbourne, VIC 3000, Australia
}

\begin{abstract}
The current-current correlation function is determined from dynamic light scattering measurements of a suspension of particles with hard sphere-like interactions. For suspensions in thermodynamic equilibrium we find scaling of the space and time variables of the current-current correlation function. This provides direct support for the notion that the motion of suspended particles can be described in terms of uncorrelated Brownian encounters. However, in the metastable fluid, at volume fractions above freezing, this scaling fails, suggesting structural impediments to momentum diffusion - the means by which particles and the suspending liquid exchange thermal energy.
\end{abstract}

Suspensions of (nearly) identical spheres have turned out to be valuable experimental model systems for exploring dynamical properties of condensed matter, particularly the dynamics of the first order, freezing-melting, transition and the glass transition. The attraction for the experimentalist lies in the sluggish motions of the suspended particles, motions that are slow enough to be tracked in real time. Theoretical analyses are almost invariably predicated on the Smoluchowski description, a description in which the suspending fluid is treated as a fluctuating hydrodynamic continuum and the typical decay time of the correlation functions of particle velocities is presumed to be much less than the typical decay time of correlation functions of their positions $[1,2]$. In other words, on time scales typical of observations by optical microscopy or spectroscopy, particle movements are assumed to be statistically independent of their momenta. More generally, this assumption, invariably underpins theoretical considerations of the dynamical properties of soft matter and biological materials [3]. Nevertheless, doubts have been raised about its validity for concentrated suspensions in particular since, as a consequence of momentum conservation, memory of the particle's velocity decays algebraically rather than exponentially [4]. Moreover, no experiment to date appears to have confirmed directly this time scale separation or established limits on its validity.

The dynamics of dense fluids or concentrated suspensions are generally pictured in terms of the cage effect - the transient localization of particles by their neighbors. The increasing persistence of this localization, consequent on increasing the density, is manifested by stretching and, at very high density, the appearance and lengthening of a plateau in the time auto-correlation function of the particle number density (the intermediate scattering function, or ISF) [5]. Another aspect of the dynamics, best exposed by the current-current correlation function (defined below), is backflow; a particle current in one direction must be compensated by a current in the opposite direction, as dictated by conservation of number density. However, this aspect of the cooperation among particles in dense fluids has been rarely considered. Yet, as we show below, this quantity exposes significant new insights. In particular, we observe scaling of currentcurrent correlation function for suspensions of particles with hard sphere-like interactions, which provides direct support for the Smoluchowski description so long as the suspension is in thermodynamic equilibrium. However, deviations from this scaling, found for the metastable, or "under-cooled", suspension, are inconsistent with the Smoluchowski description.

The colloidal particles used in these experiments consist of cores of a copolymer of methylmethacrylate and trifluoroethylacrylate [6]. To prevent coagulation the particles are coated with a thin $(\approx 10 \mathrm{~nm})$ layer of poly12-hydroxystearic acid. Their suspension in cis-decalin reduces the samples' turbidity sufficiently that multiple scattering can be neglected irrespective of the particle concentration. The particles' average hydrodynamic radius and polydispersity, determined by a combination of static and dynamic light scattering are $\mathrm{R}=185 \mathrm{~nm}$ and $8 \%$, respectively [7]. The lowest volume fraction, $\varphi$, where separation of the colloidal crystal phase is observed [8] is identified with the freezing point, $\varphi \mathrm{f}=0.494$, known for the perfect system of hard spheres [9]. Between $\varphi f$ and the melting volume fraction, $\varphi \mathrm{m}=0.545$, there is a coexistence between crystalline and fluid phases [9]. Size polydispersity effectively delays nucleation of colloidal crystals sufficiently to allow the metastable state to be studied [10]. A glass transition is found at $\varphi g \approx 0.57$ $[8,11]$. Previous works give details of sample preparation [6], equilibrium phase separation [12] and dynamic light scattering (DLS) protocols [5]. For the results presented here, experiments of 1000 s duration were made on an ALV 6010 Fast DLS spectrometer. To obtain statistically valid averages, 50 independent runs were made at each scattering angle.

The basic dynamical property obtained by DLS is the coherent intermediate scattering function (ISF), or normalized auto-correlation function of the qth spatial Fourier component, $\delta \rho(q, \mathrm{t})$ of the particle number density fluctuations [1], 


$$
f(q, \tau)=1+\frac{\langle I\rangle_{T}}{\langle I\rangle_{E}}\left\{\left[\frac{\langle I(0) I(\tau)\rangle_{T}-\left\langle I^{2}\right\rangle_{T}}{\langle I\rangle_{T}^{2}}+1\right]^{\frac{1}{2}}-1\right\}
$$

Here $\tau$ is the delay time, "*" denotes complex conjugation and the angular brackets denote the ensemble average. The current-current correlation function [11],

$$
f(q, \infty)=1+\frac{\langle I\rangle_{T}}{\langle I\rangle_{E}}\left\{\left[2-\frac{\left\langle I^{2}\right\rangle_{T}}{\langle I\rangle_{T}^{2}}\right]^{\frac{1}{2}}-1\right\}
$$

is obtained by numerically differentiating the ISF. In the results below delay times are expressed in units of the Brownian time, $\tau \mathrm{B}=\mathrm{R} 2 /(6 \mathrm{Do})(=0.13 \mathrm{~s})$, where Do is the diffusion constant for freely diffusing particles, and all lengths (including 1/q) are expressed in units of the particle radius.

Where it can be discerned from experimental and numerical noise, $\mathrm{C}(\mathrm{q}, \tau)$ is negative over the time window, $10-3<\tau<104$ approximately. Absolute values are shown in Fig. 1 for several volume fractions, two below $\varphi$ f and one above, and wavevevectors, $1.0 \leq \mathrm{q} \leq 4.9$, bracketing the position, $\mathrm{qm}^{\sim} 3.5$, of the main peak of the static structure factor. The fact that, like the velocity auto-correlation function [14], $\mathrm{C}(\mathrm{q}, \tau)$ is negative indicates that, in the present time window, the particle currents are dominated by conservation of particle number - transport of particles in one direction must be compensated by transport in the opposite direction - rather than conservation of momentum which tends to maintain the direction of movement.

In the hypothetical case of diffusive density fluctuations, $F(q, \tau)=\exp \left[-q^{2} D \tau\right]$. and $C(q, \tau)=$ $-q^{4} D^{2} \exp \left[-q^{2} D \tau\right]$. Then the quantity $C(q, \tau) /\left(q^{2} D\right)^{2}$ would be an exponential function of $q^{2} D \tau$ independent of q. A finite concentration of particles incurs not only excluded volume effects among them but also confines the spatial distribution of hydrodynamic modes (ie, momentum currents) in the suspending liquid. Assuming the hydrodynamic modes propagate instantaneously their spatial distribution can be characterized by the short-time diffusion coefficient [1],

$$
f(q, \infty)=1+\frac{\langle I\rangle_{T}}{\langle I\rangle_{E}}\left\{\left[2-\frac{\left\langle I^{2}\right\rangle_{T}}{\langle I\rangle_{T}^{2}}\right]^{\frac{1}{2}}-1\right\}
$$

where $\tau_{\ell}$ refers to the lower limit of the experimental time window. In the present context one may consider q4Ds2(q), shown in Fig. 2, as the contribution to the amplitude of the current-current correlation function due to those hydrodynamic modes in the suspending liquid

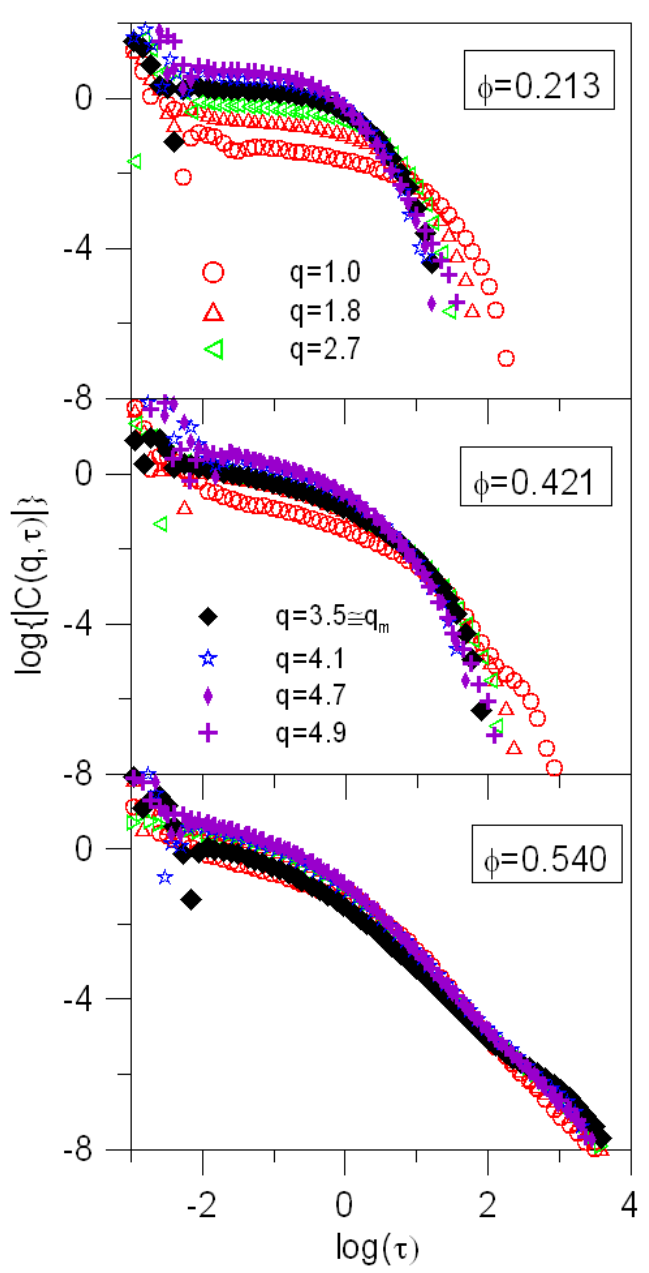

Figure 1: Fig. 1. (Color on-line) Double logarithm plots of the absolute value of the current-current correlation function, $\mathrm{C}(\mathrm{q}, \tau)$ versus delay time for volume fractions and wavevectors indicated.

that propagate instantaneously. At all but the lowest wavevectors this contribution decreases with increasing $\varphi$.

Fig. 3 shows the current-current correlation function normalized by $\mathrm{q} 4 \mathrm{Ds} 2(\mathrm{q})$ and the delay time scaled as $\mathrm{q} 2 \mathrm{Ds}(\mathrm{q}) \tau=\tau^{*}$. Representative data are shown for two volume fractions below of and one above. For the two volume fractions below $\varphi f$, the result, $\mathrm{C}^{*}\left(\mathrm{q}, \tau^{*}\right)=\mathrm{C}(\mathrm{q}, \mathrm{q} 2 \mathrm{Ds}(\mathrm{q}) \tau) / \mathrm{q} 4 \operatorname{Ds} 2(\mathrm{q})$, shows no systematic variation with q. It can be described by a stretched exponential function, $\mathrm{C}^{*}\left(\mathrm{q}, \tau^{*}\right)=-\mathrm{A} \exp \left[-\left(\tau^{*} / \tau \mathrm{x}\right) \gamma\right]$, of the scaled delay time. The stretched exponential fits to the data for the full range of volume fractions are shown in Fig. 4, with the fit parameters shown in the inset. Experimental noise accords considerable latitude to the parameters, as indicated by the error bars on the stretching index $\gamma$. Nonetheless, as $\varphi$ increases from zero to 0.5 , one sees that $\gamma$ decreases from approximately one and A increases from zero ie, with increasing volume frac- 


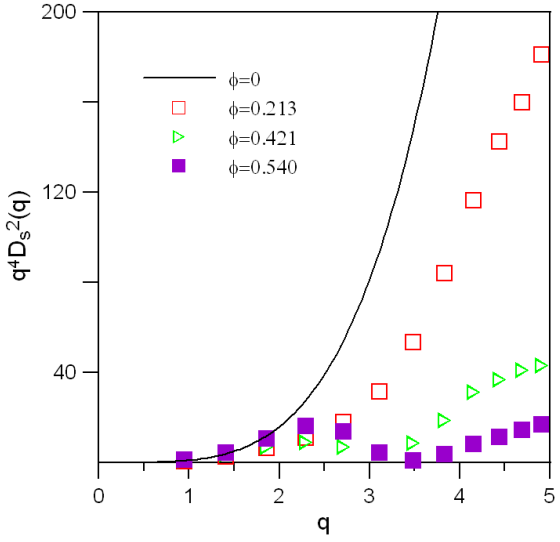

Figure 2: Fig. 2. (Color on-line) q4Ds2(q) versus q for volume fractions indicated. [Note again that $\mathrm{q}$ is scaled by the particle radius.]

tion backflow becomes more retarded $(\gamma$ decreases) and stronger (A increases). In contrast the stretching index of the VAF is independent of $\varphi$ [12]. The concomitant decrease in $\tau x$, we suggest, reflects the decrease in the (average) size of the neighbor cages and the consequent increase in the rate with which the directions of the particles' motions within those cages change.

The scaling, or factorization of space and time variables, of $\mathrm{C}(\mathrm{q}, \tau)$, seen in Fig. 3, merely demonstrates that excluded volume effects among the particles causes localization of the particle current. Indeed, time correlation of the current is synonymous with its localization in space. The strength of such localization, or correlation, expressed by the value of A (Fig. 4 (inset)), increases with increasing volume fraction from zero, at $\varphi=0$, to approximately 0.8 at $\varphi$.

These inferences rest on the accuracy of the approximation that regards the fastest number density fluctuations, detected in these experiments, as diffusive. Determination of the relevant diffusion coefficient, Ds(q) [15], by fitting an exponential function to the initial decay of the ISF, does not in itself imply that those processes are diffusive and that, therefore, all memory of the disturbances imparted to the particles by hydrodynamic modes in the suspending liquid is lost. However, the observation that $\mathrm{C}^{*}\left(\mathrm{q}, \tau^{*}\right)$ is independent of $\mathrm{q}$ indicates that in the present time window $(\tau>)$ particle currents can be considered from the perspective of instantaneously propagating hydrodynamic modes alone. In other words the scaling of $\mathrm{C}(\mathrm{q}, \tau)$ vindicates the assumption that underpins the Smoluchowski equation $[1,2,4]$.

However appealing this result may be, it is necessarily an approximation. This approximation is only valid in that time window where, for sufficiently large and heavy particles in a sufficiently viscous fluid, the constraints imposed by conservation of number density dominate those imposed by conservation of momentum. Then, as is ev-

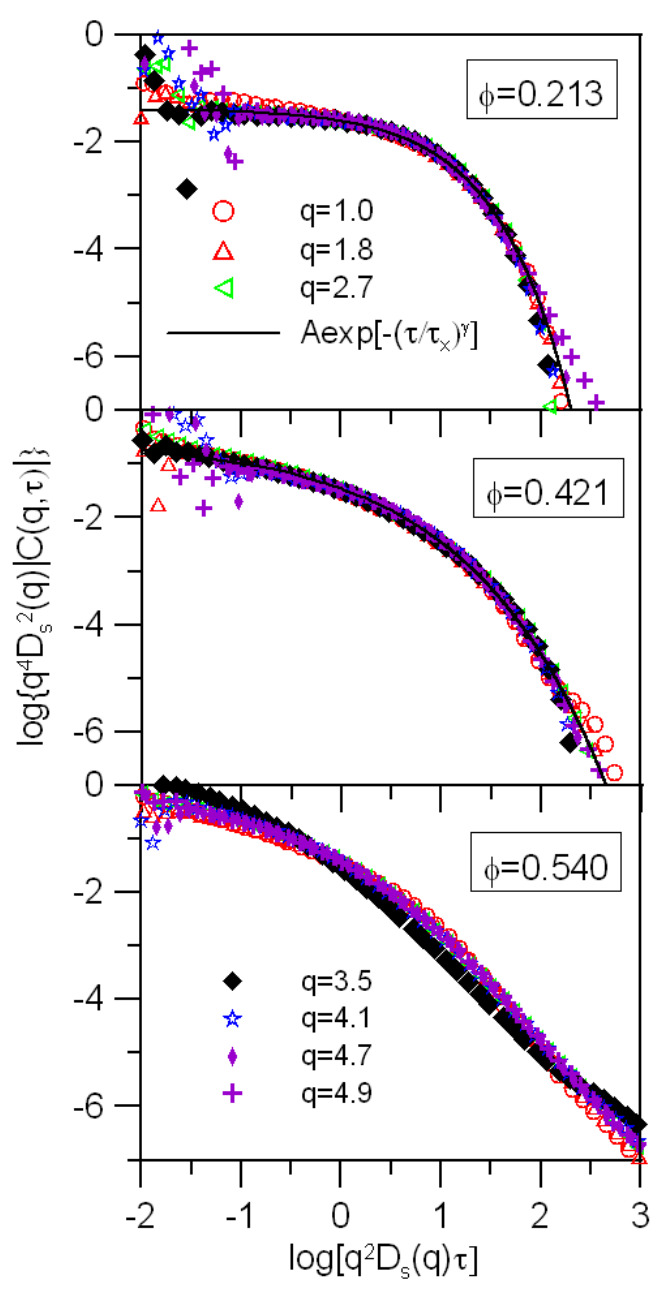

Figure 3: Fig. 3. (Color on-line) Double logarithm plots of the current-current correlation function normalized by the factor q4Ds2(q) (shown in Fig. 2) versus scaled delay time $\mathrm{q} 2 \operatorname{Ds}(\mathrm{q}) \tau$, for volume fractions and wavevectors indicated.

idently the case in this study, the decay of time correlations of the particle number density and their currents to the experimental noise floor can be presumed to occur through un-correlated Brownian encounters. Conservation of momentum must prevail, however, for very small and very large delay times. While the stretched exponential, seen here for the current-current correlation function and in Ref. 12 for the VAF, may well dominate in the experimental time window $(<\tau<\tau \max \approx 104)$, the algebraic, $\tau-3 / 2$ hydrodynamic "tail", that manifests momentum conservation [14], must dominate for very small $(\tau<<)$ and very large $(\tau>>\tau \max )$ delay times.

For $\varphi=0.54$ (Fig. 3c) there are qualitative variations with q-scaling is not possible. Similar behaviour is observed for all volume fractions above $\varphi$. One sees for $\mathrm{q}=3.5(\sim \mathrm{qm})$, in particular, that the slope of $\mathrm{C}^{*}\left(\mathrm{q}, \tau^{*}\right)$ is non-monotonic. To better expose the inflection that appears to have developed we show, in Fig. 5, the logarithmic derivative, , for several volume fractions. For $\varphi<\varphi$, 


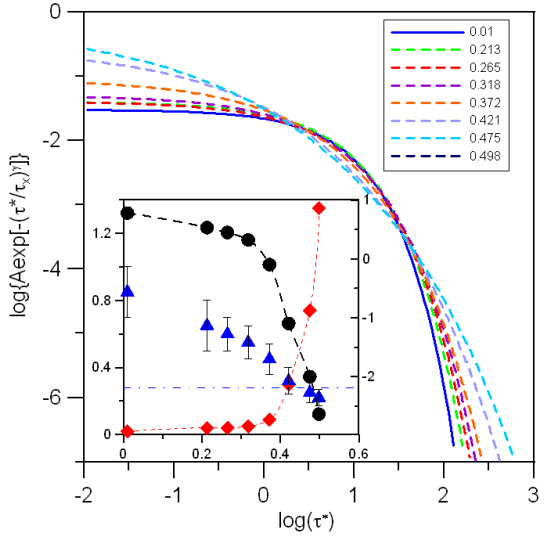

Figure 4: Fig. 4. (Color on-line) Stretched exponential fits to scaled current-current correlators verses scaled delay time for volume fractions increasing from bottom to top. Inset shows parameters verses volume fraction: A - Diamonds, left axis; $\gamma$ - triangles, left axis; $\log (\tau \mathrm{x})$ - circles, right axis. The horizontal dashed-dot line is the value of the stretching index of the VAF [12]. The other dashed lines are drawn to guide the eye.

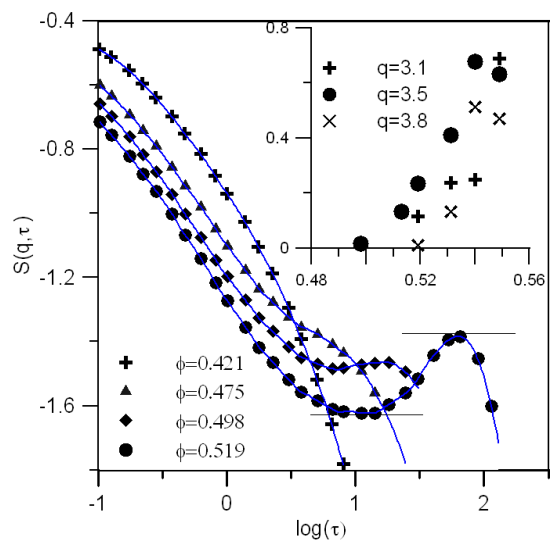

Figure 5: Fig. 5.(Color on-line) Logarithmic derivative, , of the current-current correlation function versus logarithm of delay time for volume fractions indicated. The lines are drawn to guide the eye. The inset shows the difference between the maximum and the minimum of $\mathrm{S}(\mathrm{q}, \tau)$ versus volume fraction for wavevectors indicated.
$\mathrm{S}(\mathrm{q}, \tau)$ decays monotonically while for $\varphi>\varphi$ f it does not. We quantify this non-monotonicity with the difference, , shown in the inset of Fig. 5. From this it appears that the impediment to the decay of the current-current correlation function signified by the non-monotonicity, whatever its nature, first sets in at $\varphi$ f for wavevectors around $q m$. For $\mathrm{q} \neq \mathrm{qm}$ it sets in at higher $\varphi$.

Previous works, on both Newtonian [15] and colloidal [12] hard spheres, have exposed the emergence, at $\varphi f$, of negative algebraic decays in the velocity auto-correlation function. This is a feature reminiscent of flow in channels [16] and porous media [17] where the fluid is presented with a structural impediment that must be accommodated by the momentum currents. As a consequence, overdamped compression waves are excited. In view of these studies we propose that any impediment to structural relaxation, such as that inferred here from the current-current correlation function, for the metastable suspension, and similarly inferred from analyses of number density fluctuations in terms of the mean-squared displacements [20], will effect viscous coupling among the particles. Of course such viscous coupling means that the notion of a short-time diffusion coefficient loses meaning.

Conclusion; Factorization of space and time variables of the current-current correlation function, observed for a hard sphere suspension in thermodynamic equilibrium, provides direct evidence to support the assumption of statistical orthogonality of momentum and configuration spaces, the assumption germane to theories of Brownian motion. This support does not extend to the metastable case, ie, for suspension volume fractions in excess of the first order freezing transition. In this case there are structural impediments to the diffusive momentum currents by which the particles dissipate their instantaneous thermal energy. 